$62^{\text {ème }}$ Congrès de la SFCO, 02024 (2014)

DOI: $10.1051 /$ sfco/20146202024

(C) Owned by the authors, published by EDP Sciences, 2014

\title{
Les foyers infectieux bucco-dentaires et les infarctus cérébraux : Méta-analyse des études de cohorte
}

\author{
Lafon A ${ }^{5,1}$, Pereira $B^{3}$, Dufour $\mathrm{T}^{6}$, Ahossi V ${ }^{5}$, Béjot $\mathrm{Y}^{4}$, Lefevre B ${ }^{1}$, Tubert $\mathrm{S}^{2}$ \\ 1 Unité fonctionnelle de Chirurgie buccale, Pôle d'Odontologie, Hôpital Maison blanche, 45 rue Cognacq-Jay, Reims, FRANCE \\ 2 Université d'Auvergne, CROC-EA4847, Centre de Recherche en Odontologie Clinique, BP 10448, F-63000, Clermont-Ferrand, FRANCE \\ 3 CHU Clermont-Ferrand, Service d'Odontologie, Hotel-Dieu, F-63000 Clermont-Ferrand, FRANCE \\ 4 Dijon Stroke Registry, EA4184, Medical School and University Hospital of Dijon, University of Burgundy, 7 boulevard Jeanne d'Arc - 21000 Dijon, FRANCE \\ 5 CHU Dijon, Service Odontologie , Hôpital Général, 3, rue Faubourg Raines, 21000 Dijon, FRANCE \\ 6 Rennes University, University of Bretagne, 1 Bât 15-2, Avenue Léon Bernard 35043 Rennes Cedex, U.F. Parodontologie, Pôle d'Odontologie et de Chirurgie Orale, CHU de Rennes, 2 place \\ Pasteur, F-35000, Rennes, FRANCE
}

Les pathologies liées à l'athérosclérose telles que les infarctus du myocarde ou les infarctus cérébraux (IC) sont les premières causes de mortalité dans nos pays occidentaux (Bèjot 2013). Au vu de l'absence de consensus et de l'absence de revue synthétique étudiant la relation des IC et la présence de foyers infectieux bucco-dentaires (FIBD) et, afin d'évaluer précisément le lien entre les FIBD et la survenue IC, une méta-analyse est réalisée.

\section{Méthode}

Les études épidémiologiques de cohorte (prospectives et rétrospectives) évaluant les effets de la maladie parodontale et de la perte dentaire sur le risque de survenue des IC ont été incluses. Sur les 743 articles identifiés, seulement 9 articles (8 études prospectives et 1 rétrospective), ont été retenus. Une analyse par méta-régression a permis de regrouper les résultats d'études comparables en compilant les risques relatifs (RR) et les hasards ratios (HR). Les résultats ont été séparés selon le type d'exposition à l'inflammation buccale choisi (maladie parodontale, gingivite, perte dentaire) et selon le critère de jugement retenu (AVC hémorragiques ou infarctus cérébral).

\section{Résultats}

Les résultats de la méta-analyse diffèrent significativement selon l'exposition d'inflammation buccale choisi et selon que I'AVC soit d'origine ischémique (IC) ou hémorragique. Les résultats montrent que le risque de survenue d'un IC est significativement augmenté avec l'existence d'une parodontite sévère (RR $1.38[1.10,1.67] p=0.001)$.
La perte dentaire semble représenter un facteur de risque majeur pour la survenue des IC (RR $1.28[1.01,1.57] p=0.001)$. Concernant la gingivite, le risque de survenu d'un IC et/ou hémorragiques n'est pas significativement augmenté (RR $1.13[0.84,1.42] \mathrm{p}=0.001)$.

\section{Conclusions}

Les résultats de cette méta-analyse montrent que la maladie parodontale est associée à la survenue des IC, confortant ainsi les hypothèses émises concernant le rôle de l'inflammation buccale dans leur survenue (Lee 2013, D’Auito 2013). La cohérence des données issues de différentes études de cohorte indique que la relation existant entre les IC et la maladie parodontale ne peut pas être uniquement expliquée par l'effet des cofacteurs communs tels que l'hypertension, le diabète, l'âge ou le tabac. La maladie parodontale semble représenter un facteur de risque indépendant de la survenue des IC.
LAFON Arnaud lafon.arn@gmail.com

This is an Open Access article distributed under the terms of the Creative Commons Attribution License 4.0, which permits unrestricted use, distribution, and reproduction in any medium, provided the original work is properly cited. 